\title{
The Relation of Golden Ratio, Mathematics and Aesthetics
}

\author{
Gyan Bahadur Thapa ${ }^{1}$, Rena Thapa ${ }^{2}$ \\ ${ }^{1}$ Department of Science and Humanities, Pulchowk Campus, IOE, Tribhuvan University, Nepal \\ ${ }^{2}$ Fine Art College, Bhotahity, Tribhuvan University, Kathmandu, Nepal \\ Corresponding author: thapagbt@ioe.edu.np
}

Received: Dec 5, 2017 Revised: Feb 15, 2018 Accepted: Feb 20, 2018

\begin{abstract}
The Golden Ratio, mathematics and aesthetics are intricately related among each other. In this paper, we exhibit the presence of mathematics in aesthetic impression that appears in nature, classic art, architecture, logo design and much more. The divine proportion can be found in music, poetry and other forms of art, however our focus here is only in the visual ones. The Golden ratio is considered sacred due to its relationship to nature and even the construction of the universe and the human body. It has been used for centuries in the construction of architectural masterpieces by the great artists, who, being able to see its beauty used it in their designs and compositions. We explain how the applications of the Golden ratio in architectures, paintings and geometrical shapes create the mystery of beauty. Further we present the existence of the divine proportion in human body and natural flora and fauna. There are a diverse number of directions, paths and tangents to which the study of this beautiful concept could take us. Besides mathematicians and artists, we expect that this paper will be interesting for general readers as well.
\end{abstract}

Keywords: Golden ratio, Fibonacci sequence, aesthetics, architecture, phyllotaxis

\section{Introduction}

Mathematics and art are considered as two distinct disciplines. Art is associated with aesthetics and mathematics is supposed to be a complete curt and dry subject; the two disciplines as two extremes. However, both mathematics and fine art are the output of human consciousness that strive to express not only the physical reality but also the metaphysical one. Reflecting on the structure of reality, artists express the psychological mood in their creation. As painting represents figurative aspect of concrete object, mathematician tries to extract specific mathematical property to investigate physical phenomenon. Mathematics and painting are related in several ways. Mathematical geometry is used to analyze paintings (both figurative and abstract) and architect in terms of shapes such as points and lines, circles, triangles, cubes etc. Likewise mathematics can be used in a more concrete direct way to analyze painting such as "the use of concepts like fractals for the comprehension of abstract paintings. . . Jackson Pollock's drip paintings in terms of fractal geometry" [4]. Mathematics and visual arts share common 
aspects in both form and function. One of such relations is explored as Golden ratio. Though Greeks in ancient period defined and formulated the Golden proportion, the celebration and the use of this mathematical phenomenon in art and architecture extends to the present day. Examples of Golden section can be seen throughout nature such as in shells, plants, flowers and animals. It is considered as the strongest and the oldest ties between mathematics and visual arts. The intriguing mathematical phenomenon employed in art, architect, music science and philosophy has aesthetic impression.

As Truth, Beauty and Goodness are interrelated, an artist searches for the Truth in the Beauty, and a scientist searches for the Beauty in the Truth. With the evolution of civilization, human beings have aspired for beauty. And the flip side of beauty is goodness which tempts human heart to yearn and explore beauty in creation. Ancient Greeks developed the science of aesthetics as a way to analyze beauty, believing that harmony is its basis. Harmony is acquired by arranging the parts, generally different in nature, by certain perfect ratio so that they meet one another creating beauty. It encompasses everything that is made by nature that creation is not aloof from the law of harmony. The term "proportion" is more relevantly used to compare relation between parts of things or to describe harmonious relation between different parts. When a formula could be found, then the loveliness of a chamomile flower and a naked body could be measured and compared. Even it is possible to compare the beauty of a sculpture, a temple, a picture, a symphony, a poem or a nocturne accordingly. There are many well-known "formulas of beauty" such as certain geometrical shapes: square, circle, isosceles triangle, and pyramid. However, the most wide-spread criterion of beauty is one unique mathematical proportion called the Golden ratio which is termed as Divine proportion or Golden section or Golden number or Golden mean. Examples of well known works, which exhibit this proportion, are Khufu's Pyramid of Egypt, the Parthenon in Athens, Greek sculpture, the "Mona Lisa" by Leonardo da Vinci, paintings by Rafael, Shishkin and the modern Russian artist Konstantin Vasiljev, music of Beethoven and Mozart etc.

The rest of the paper comprises the sections that include concept and history of Golden ratio, quadratic formula and algebraic derivation of golden number, geometrical golden shapes, golden section in nature, human body, art, architecture and conclusion.

\section{Golden Ratio and its History}

The Golden Ratio is an irrational number that is approximately equal to 1.618 , which is represented by the Greek symbol known as phi $(\phi)$. The digits after decimal in the Golden ratio just keep on going and never end as such $1.61803398874989484820 \ldots \ldots$ When the ratio is used in cubic geometry, it is called the Golden section. The Golden rectangle refers to a rectangle with a short to long side ratio of 1: 1.618. An interesting aspect of the Golden rectangle is that if one cuts out a square starting from one of the short sides of the Golden rectangle one will have another Golden rectangle [1]. When an isosceles triangle has the ratio of the perpendicular $a$ to the base $b$ in the Golden ratio, it is called a Golden triangle. Similarly, there is the Golden spiral which grows logarithmically. It is important to know that the geometrical shapes of the rectangle, 
triangle and spiral get their "Golden" name when they have properties that connect them back to the Golden ratio.

The concept of Golden ratio has close connection to the Fibonacci sequence which is named after Leonardo Fibonacci, the great mathematician. This sequence begins with the numbers 1 and 1 . In order to get the next number, one must add the previous two numbers in the sequence as follows:

$$
1+1=2,1+2=3,2+3=5,3+5=8,5+8=13,8+13=21, \ldots
$$

Therefore, the pattern of Fibonacci sequence is: $1,1,2,3,5,8,13,21,34,55, \ldots \ldots$ where the Golden ratio ties into this sequence through the ratio between the numbers. This can be seen in the following:

$$
\begin{array}{lc}
2 / 1=2.0 & \multicolumn{1}{c}{3 / 2=1.5} \\
5 / 3=1.67 & 8 / 5=1.6 \\
13 / 8=1.625 & 21 / 13=1.615 \\
34 / 21=1.619 & 55 / 34=1.618 \\
89 / 55=1.618 & \ldots \ldots \ldots \ldots
\end{array}
$$

Narain [6] states on The Golden Ratio Page, as the numbers in the sequence get larger; the ratio will eventually become the same number, which is the Golden Ratio. "One more interesting thing about $P h i$ is that it is reciprocal. If you take the ratio of any number in the Fibonacci sequence to the next number, the ratio will approach the approximation of 0.618 . This is the reciprocal of Phi: $1 / 1.618=0.618$ " [6]. There is not another known number to have this property outside the Fibonacci sequence. The idea behind the Golden ratio is: if a line is divided into two parts, the ratio of longer part and smaller part should be equal to the ratio of whole length and longer part. This makes the Golden ratio as in Fig. 1.
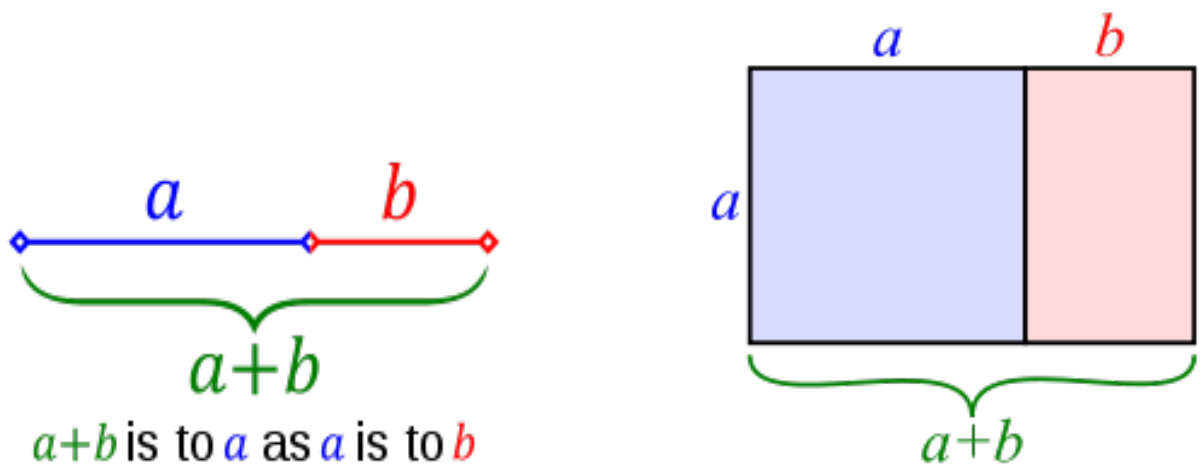

Fig. 1: Golden Ratio (http://www.mathsisfun.com/numbers/golden-ratio.html)

More precisely, we have

$$
\frac{a+b}{a}=\frac{a}{b}
$$


Equivalently, they are in the golden ratio if the ratio of the larger one to the smaller one equals the ratio of the smaller one to their difference as follows:

$$
\frac{a}{b}=\frac{b}{a-b} \text {. }
$$

After simple algebraic manipulations, i.e., multiplying equation (1) by $a / b$, or equation (2) by $(a-b) / b$, both of these equations are equivalent to

$$
\left(\frac{a}{b}\right)^{2}=\frac{a}{b}+1
$$

which is the quadratic expression, and hence the Golden Ratio is

$$
\frac{a}{b}=\phi \text {. }
$$

It is believed that the Egyptians were well aware of it and used in building pyramids and later they taught this concept to Greeks. So the ratio is very connected to the Greeks as it was named after Phidia, the sculptor of Athena, who made most of the decoration on Parthenon.

Legend says the Golden ratio was discovered by Pythagoras and that it was through him that the true knowledge of this ratio began to be understood. It is believed that the Greek Philosopher Pythagoras discovered the concept of harmony while listening to the different sounds produced as the blacksmiths' hammers hit their anvils. After further studies using stringed instruments and observing nature, he came to the conclusion that the ratio of small integers defines beauty. "With this discovery, the Pythagoreans saw the essence of the cosmos as numbers and numbers took on special meaning and significance. The symbol of the Pythagorean brotherhood was the pentagram in itself embodying several Golden means" [7]. Euclid, a Greek mathematician who is known as the Father of Geometry, was the first person to write a definition of the Golden ratio: "A straight line is said to have been cut in extreme and mean ratio when, as the whole line is to the greater segment, so is the greater to the less" [2]. The "extreme and mean ratio", was not referred to as "golden" until the early 16th century AD. Luca Pacioli equated the Golden ratio with God in his book, La Divine Proportione (The Divine Proportion). Leonardo Da Vinci contributed several drawings to La Divine Proportione and made reference to the "section aurea" (Latin for "golden section"). There was a keen interest in the Divine proportion during the Renaissance among artists, architects, scientists and mystics. The idea of using the Golden ratio in art and architecture was aesthetically pleasing and was widely established during this time [9].

\section{Some Golden Shapes}

\subsection{Golden Triangle}

The Golden triangle can be characterized as an isosceles triangle $\mathrm{ABC}$ with the property that bisecting the angle $\mathrm{C}$ produces a new triangle $\mathrm{CXB}$ which is a similar triangle to the original. If angle $\mathrm{BCX}=\alpha$, then $\mathrm{XCA}=\alpha$ because of the bisection, and $\mathrm{CAB}=\alpha$ because of the similar 
triangles; $\mathrm{ABC}=2 \alpha$ from the original isosceles symmetry, and $\mathrm{BXC}=2 \alpha$ by similarity. The angles in a triangle add up to $180^{\circ}$, so $5 \alpha=180$, giving $\alpha=36^{\circ}$. So the angles of the golden triangle are thus $36^{\circ}, 72^{\circ}, 72^{\circ}$. The angles of the remaining obtuse isosceles triangle AXC (sometimes called the golden gnomon) are $36^{\circ}, 36^{\circ}, 108^{\circ}$. Suppose $\mathrm{XB}$ has length 1 and the length of $\mathrm{BC}$ be denoted by $\varphi$. Because of the isosceles triangles, we have $\mathrm{XC}=\mathrm{XA}$ and $\mathrm{BC}=$ $\mathrm{XC}$, and hence $\mathrm{XC}=\mathrm{XA}=\mathrm{BC}=\varphi$. Thus, $\mathrm{AC}=\mathrm{AB}=\varphi+1$. But triangle $\mathrm{ABC}$ is similar to triangle $\mathrm{CXB}$, so $\mathrm{AC} / \mathrm{BC}=\mathrm{BC} / \mathrm{BX}$, and hence $\mathrm{AC}=\varphi^{2}$. Thus $\varphi^{2}=\varphi+1$, confirming that $\varphi$ is indeed the Golden ratio. Similarly, the ratio of the area of the larger triangle AXC to the smaller CXB is equal to $\varphi$, while the inverse ratio is $\varphi-1$.

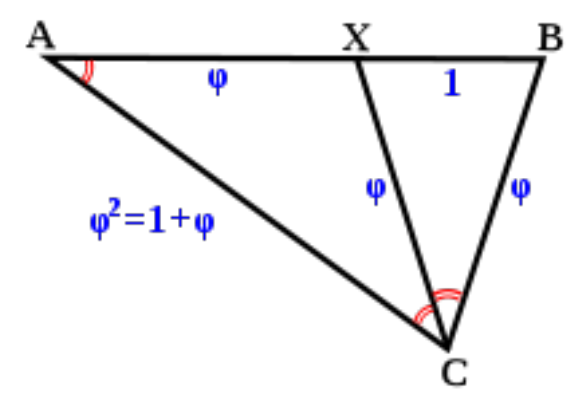

Fig. 2: Golden Triangle (http://en.wikipedia.org/wiki/file)

\subsection{Golden Rectangle}

Golden rectangle can be sketched in the following steps as shown in the Fig. 3:

Step 1: Begin with a square $A B C D$. Find the midpoint $M$ of $A B$.

Step 2: Draw a circle with radius $M C$, centered at $M$. Produce the side $A B$ through $B$ until it intersects the circle at the point $E$.

Step 3: Draw a line $E F$ perpendicular to $A E$. Produce $D C$ through $C$ up to the point $F$. The rectangles $\quad A E F D$ and $B E F C$ are known as Golden Rectangles.
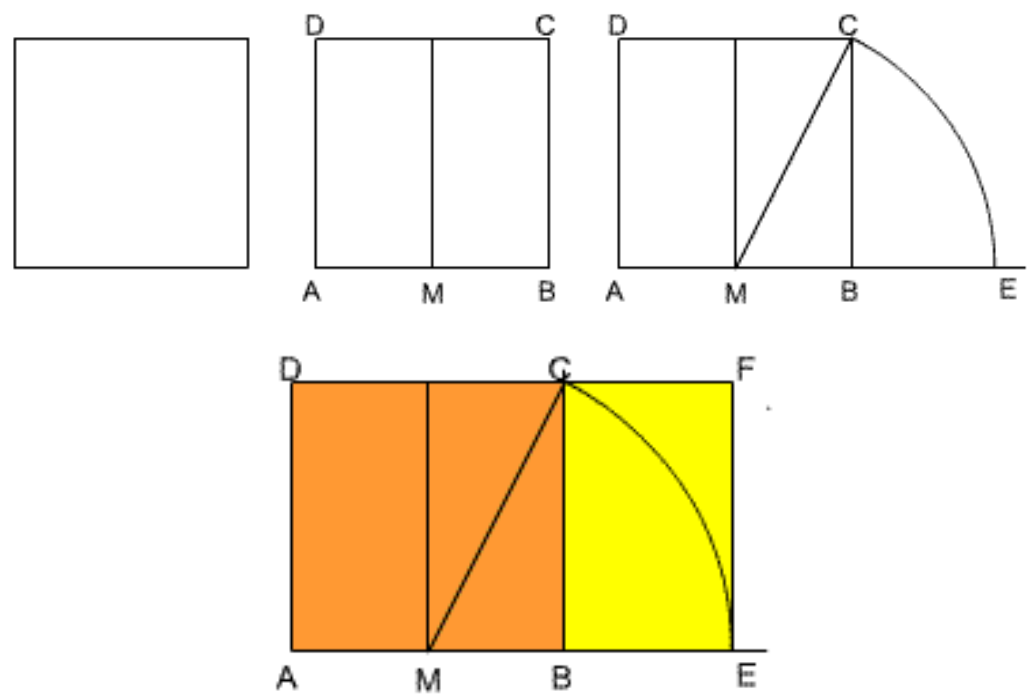

Fig. 3: Golden Rectangle (http://nrich.maths.org) 


\subsection{Golden Spiral}

Golden Spiral is drawn in the following steps as sketched in Fig. 4:

Step 1: Begin with Golden Rectangle. Construct a square by drawing a circle whose radius is the height of the Golden Rectangle.

Step 2: Repeat this process inside the smaller Golden rectangles.

Step 3: Inscribe quarter circles in each square to create the spiral.

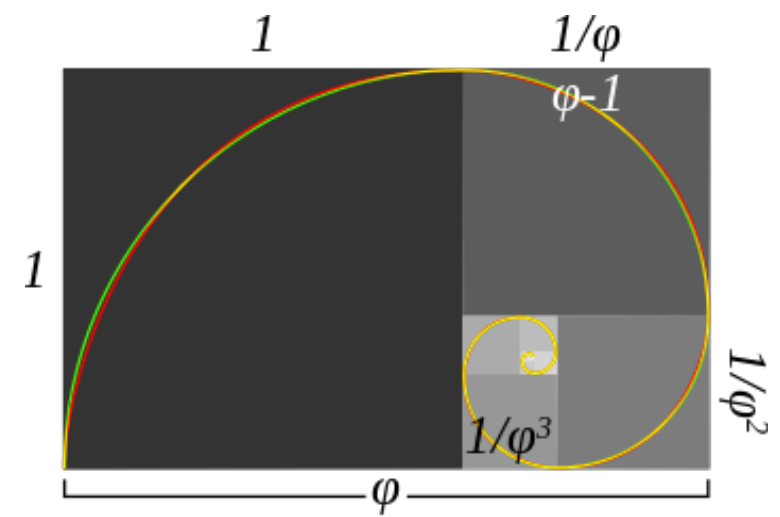

Fig. 4: Golden Spiral (http://en.wikipedia.org/wiki/file:FakeRealLogSpiral.Svg)

\subsection{Golden Pentagram}

The regular pentagon consists of a number of wonderful figures, which are widely used in works of art. The law of the "golden cup" was well known in ancient Egypt and classic Greece, which was used by architects and goldsmiths. The Pentagon and Pentagram have Golden relationships as shown in Fig. 5. The ratio of the side of a regular pentagon to its diagonal is $\varphi$. In the condition, the pentagram is inscribed within the pentagon, many of the ratios between segments are also $\varphi$. If a pentagon is divided by diagonals from one vertex, the resulting triangles are known as Golden triangles. The middle triangle is an acute Golden triangle and the other two are obtuse Golden triangles.

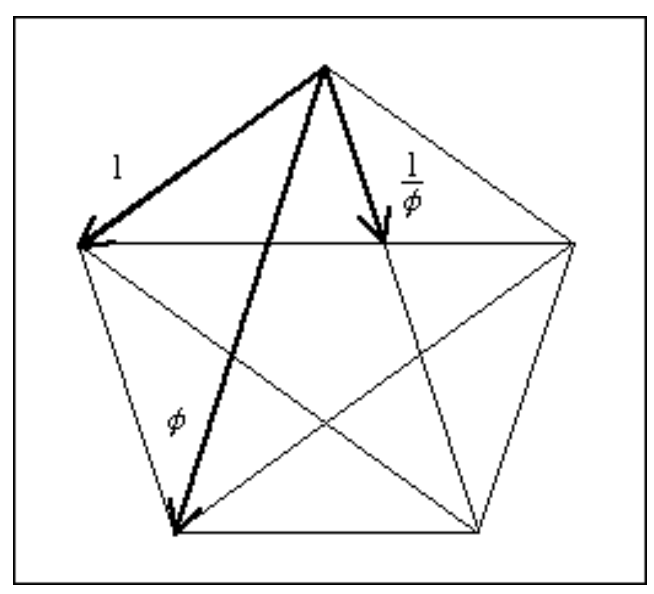

Fig. 5: Golden Pentagon and Pentagram (http://plus.maths.org/issue3/fibonacci) 


\section{Golden Ratio and Nature}

The Golden proportion as Golden spirals and Golden pentagon are commonly present in nature. Plant and animal worlds hold the abundance of Golden symmetry in their form, internal and external both. For example, animal horns grow only from one end resulting on the equiangular spiral. It is proved that among different kinds of spirals showing in horns of rams, goats, antelopes and other horned animals, the Golden spirals meet most often. The plant tendrils become twisted by spirals, the growth of tissues in tree's trunks is formed by spiral there, the sunflower seeds appear on the spirals, the helical motions are watched at growth of the roots and sprouts.
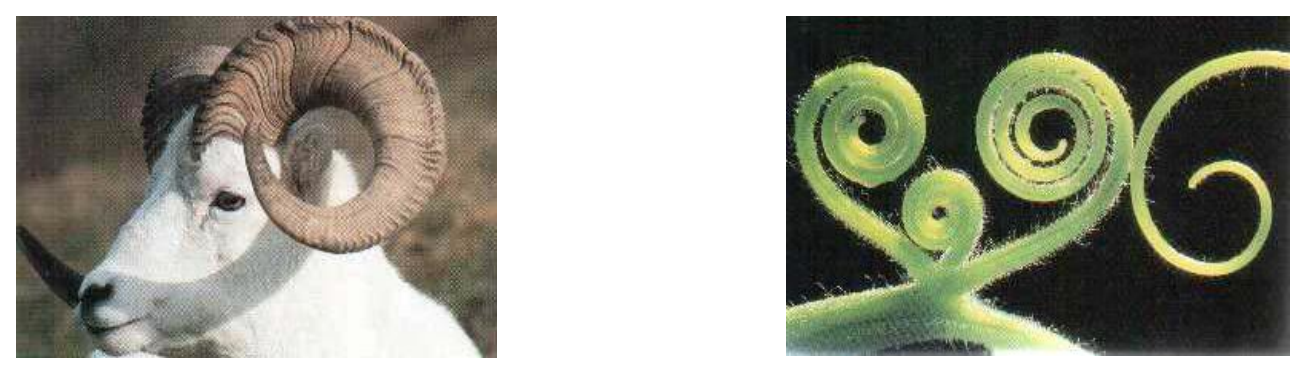

Fig. 6: The "Golden" Spirals in animal horns and plants

In the nature, the forms based on the pentagonal symmetry (marine asters, marine hedgehogs, flowers) are widespread. The flowers of water lily, wild rose, hawthorn, small nail, pear, apple, strawberry and many other flowers owe five-petal. Some of the evidences of pentagonal symmetry apparent in nature are shown in Fig. 7.

(a)

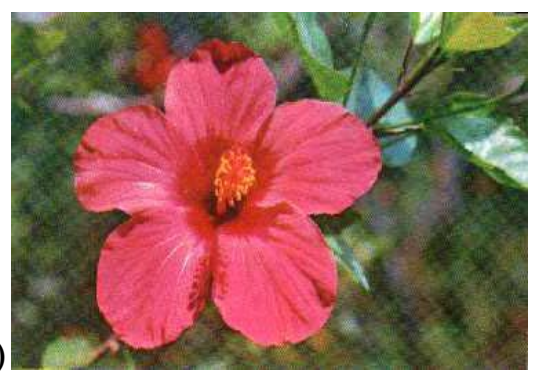

(c)

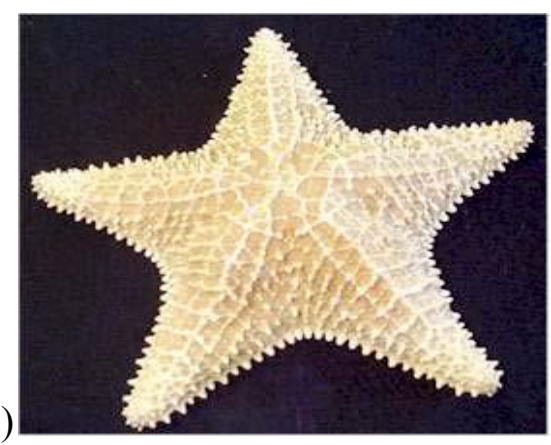

(b)

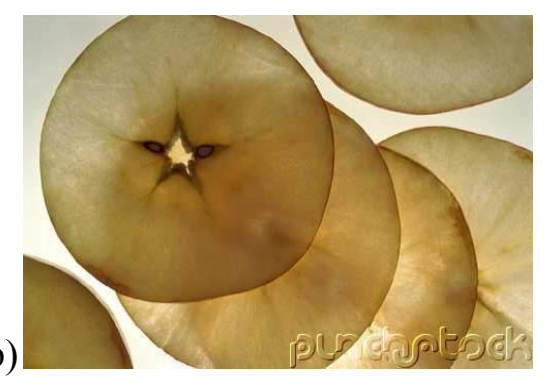

(d)

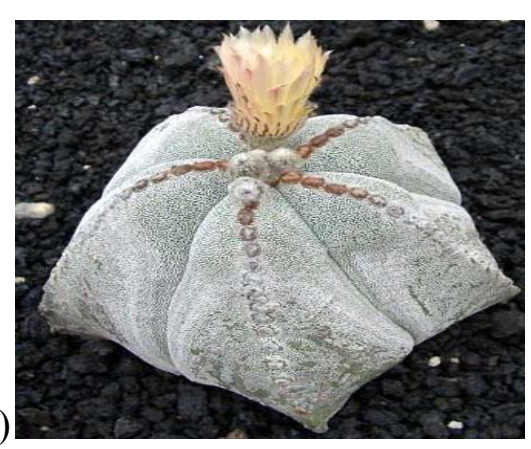

Fig. 7: Pentagonal symmetry in Nature: (a) Chine rose (b) Apple in cut (c) Marine Star (d) Cactus (http://www.goldenmuseum.com) 
The next noticeable surprise in nature is the screw symmetry in organism which is formulated as the stringent mathematical laws. The arrangement of leafs on stems of plants has a stringent mathematical structure and this phenomenon is called "phyllotaxis" in botany. The pattern of phyllotaxis exists in screw arrangement of leaves on plant stems (branches on trees, petals in racemes and so on). Botanists affirm that the fractions describing the plant screw axes form the stringent mathematical sequence consisting of the adjacent Fibonacci number ratios, i.e., 1/2, 1/3, $2 / 5,3 / 8,5 / 13,8 / 21,13 / 34, \ldots .$. . Arrangement of leaves on the plant stem conforms the phyllotaxis phenomena, particularly the screw axis of symmetry (Fig. 8). Leaves can be noticed at different altitudes of the stem along the screw curve winded round of its stem.
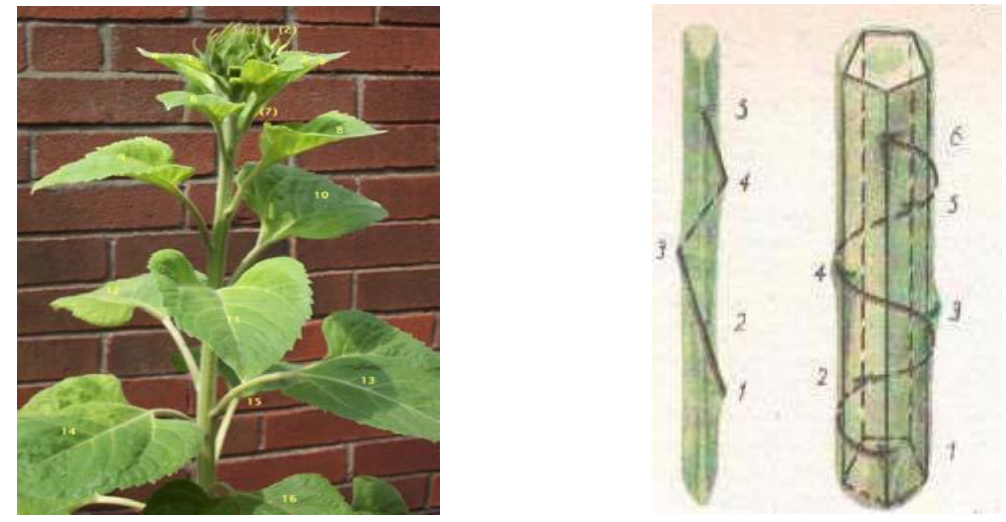

Fig. 8: The screw stem axis of symmetry (http://www.goldenmuseum.com/)

To pass from the leaf of 1 to the leaf of 2, it is necessary to turn the leaf of 1 around of the stem axis on $120^{\circ}$ counter-clockwise (if to look from below) and then to move the leaf of 1 along the stem in vertical direction so long as it will be combined with the leaf of 2 . Repeating similar operation, one needs to pass from the leaf of 2 to the leaf of 3 and so on. It is necessary to attract attention to the fact that the leaf of 4 lies above of the leaf of 1 (as though repeats it, but its level is higher). While moving from the leaf of 1 to the leaf of 4 , it turns triply on the angle $120^{\circ}$ and completes the full revolution around of the stem axis $\left(120^{\circ} \times 3=360^{\circ}\right)$. The turning angle of the screw axis is called leaf divergence angle. The revolutions around of the stem axis for transition from the lower leaf to the upper one arranged exactly above lower can be equal not only 1 , but also 2, 3 and so on. This number of the revolutions is called the leaf cycle. Botanic screw leafarrangement can be characterized with the help of some fraction; the numerator of the fraction is equal to the leaf cycle and the denominator to a number of leafs in this leaf cycle. In this case one finds the screw axis of the kind of $1 / 3$. The fraction describing the given axis is expressed by $2 / 5$; the leaf divergence angle is equal to $144^{\circ}\left(360^{\circ}: 5=72^{\circ} ; 72^{\circ} \times 2=144^{\circ}\right)$. There might be more intricate axes, for example, of the kind of $3 / 8,5 / 13$ and so on [8]. The phyllotaxis fractions from Fibonacci sequence $1,1,2,3,5,8,13,21,34,55,89, \ldots$. are characteristic of different plants. For example, the fraction of $1 / 2$ is peculiar to cereals, birch, grapes; $1 / 3$ to sedge, tulip, alder; $2 / 5$ to pear, currants, plum; $3 / 8$ to cabbage, radish, flax; 5/13 to spruce, jasmine and so on. Practically all racemes and densely packaged botanic structures (pine and cedar cones, pineapples, cactuses, heads of sunflowers and many others) also strictly follow to Fibonacci numbers regularity. 
(a)

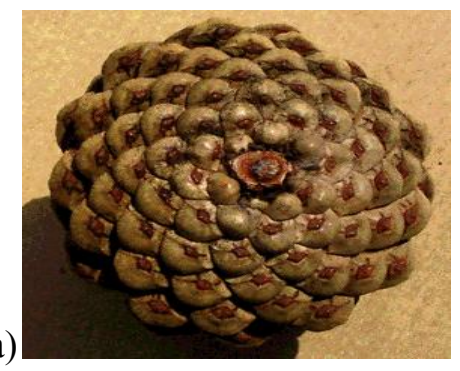

(b)

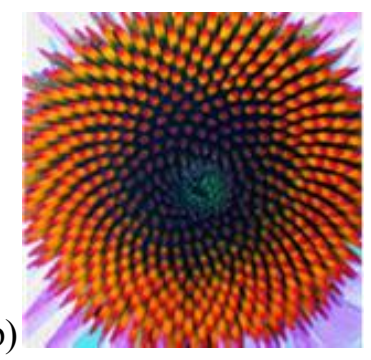

Fig. 9: Phyllotaxis Structures: (a) pine cone (b) head of sunflower (http://www.goldenmuseum.com/)

\section{Golden Ratio and Human Body}

A human body and all its parts are based on the principle of the Golden proportion. A harmonic human body is divided by the navel into the golden section. Human bodies that do not follow golden proportions look formless. The navel (belly button) represents the mean of the golden ratio. When the navel is positioned as such that the ratio of the short half to the long half is equal to the ratio of the whole to the long half, the body implies the golden ratio with perfect harmony.

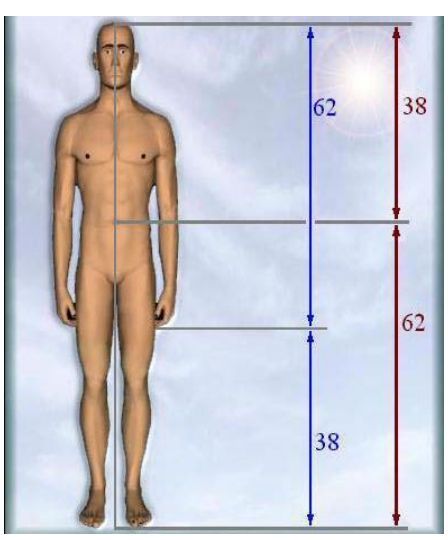

(a)

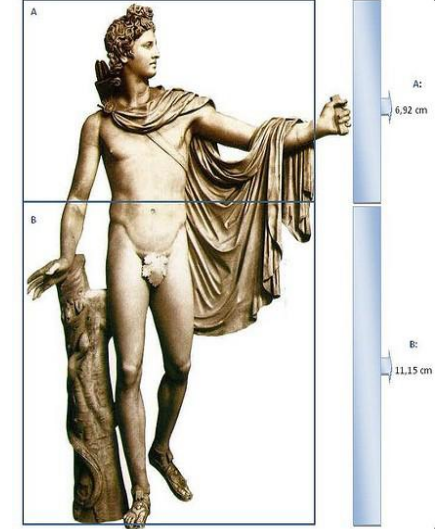

(b) sculpture of Apollo of Belvedere

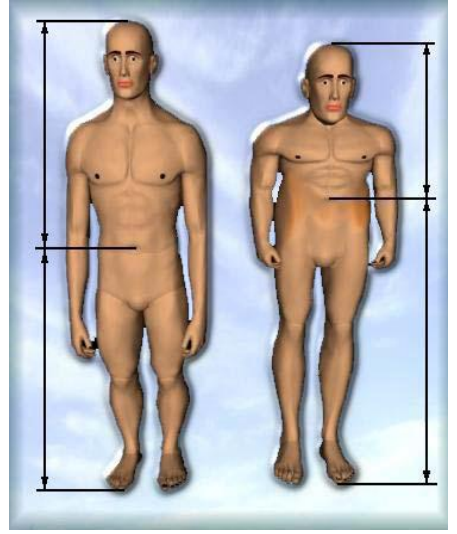

(c)

Fig. 10: Human Body: Harmony (a and b); Disharmony (c) (http://www.goldenmuseum.com/, http://goldenratio.wikidot.com/greek-and-roman-art)

Similarly the formation of human hand creates a Golden section in relation to our arm, as the ratio of forearm to our hand is also 1.618 , the Golden ratio. It is proved that the human face is based entirely on the Golden ratio. Particularly, the head forms a Golden rectangle with the eyes at its midpoint. The mouth and nose are each placed at Golden sections of the distance between the eyes and the bottom of the chin. The proportions of the length of the nose, the position of the eyes and the length of the chin, all conform to some aspect of the Golden ratio (see [8] for more detail). 


\title{
6. Golden Ratio and Architecture
}

The use of Golden ratio is prevalent in ancient and modern architecture. The creation of the goddess Athens temple, the magnificent Parthenon, is outcome of joint efforts of architects and sculptors of ancient Greece. It is rightfully considered as the greatest monument of ancient Greek architecture. Parthenon is the exclusive human creation furtive with architectural and sculptural grandeur and the main cause of Parthenon's beauty is the exquisite harmony of its parts based on the Golden ratio. Some of the features of Parthenon are described in [3] as follows:

\begin{abstract}
Analysis of the proportions of the building has revealed many hidden golden section rectangles. The ground plan consists of two reciprocal golden rectangles, and the interior chamber is in golden proportion; the main façade fits into a single golden rectangle, and a comparison of the façade with ground plan reveals the basic harmonic relationships we associate with golden proportion. All of these connections add a sense of order and continuity to sequence of space and forms.
\end{abstract}

Similarly, the divine proportion was used in the construction of the meditation symbol Sri Yantra in ancient India (Fig. 11). Sri Yantras are formed by 9 interlocking isosceles triangles, 4 of them point upwards representing the female energy Shakti, while the other 5 point downwards representing the male energy Shiva. The standard form of the Sriyantra, with the 9 interwoven triangles, constitutes a total of 43 triangles. The amazing part is that the triangle of the Yantra is a proportionate cross-section of the Giza Pyramid, incorporating both special numbers $\pi$ (3.142...) and $\phi(1.618 \ldots)$ ratio. The base angle of the triangle in the Yantra is seen to be around $51^{\circ}$, the same value that was attributed to the base of the great Pyramid of Giza.

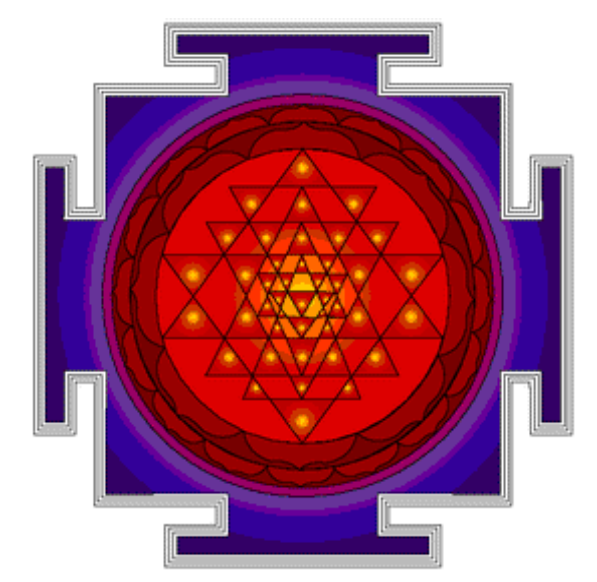

Fig. 11: Indian Meditation Symbol Sri Yantra (commons.wikimedia.org/wiki/SriYantra_construct.svg)

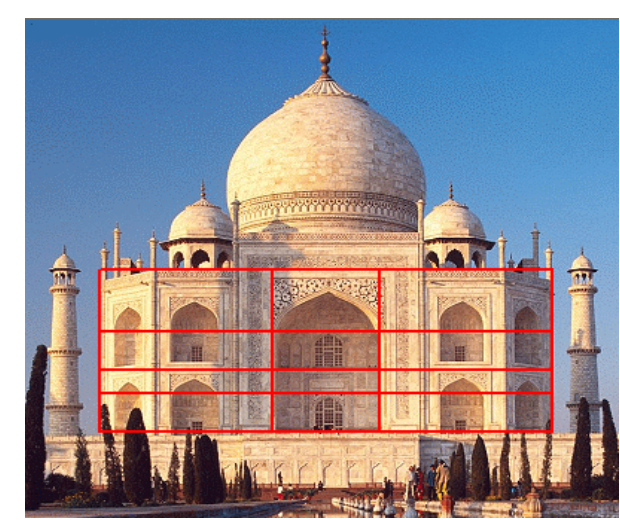

Fig. 12: Main Building of Taj Mahal (google.com/site/funwithfibonacci/taj-mahal)

Similarly, the main building of Taj Mahal is another monument in the oriental architecture employing the golden proportion. The rectangles that served as the basic outline for the exterior of the building are all in the Golden ratio (Fig. 12). The gigantic pyramids of ancient Egypt are magnificent wonders attributing the golden proportion. These huge monuments were constructed 
for preservation of Pharaoh's body and his spirit and for glorifying his authority. Egyptian pyramids demonstrate Egyptians' mathematical knowledge and their keen interest to incorporate it in pyramids before the era of Pythagoras. Among them, Khafre's pyramid is rather interesting. "The measurements of Khafre's pyramid showed that the inclination angle of the lateral faces is equal to $53^{\circ} 12^{\prime}$ that corresponds to the leg ratio of the right triangle: 4:3. Such leg ratio corresponds to the well-known right triangle with the side ratios: $3: 4: 5$; this one is called perfect, sacred or Egyptian triangle" [8].

\section{Golden Ratio and Art}

While exploring the masterpieces of world arts, critics have found the wide use of the proportion of the Golden section in the compositional structures of Michelangelo (pentagram), Rafael Santi (Golden triangle), Iva Shishkin, Konstantin Vasil'ev (Golden rectangle). The Golden ratio is prevalent in Da Vinci's The Annunciation, Madonna with Child and Saints, The Mona Lisa and St. Jerome. He was famous for using the Golden ratio in his works. The Mona Lisa, a well known portrait of a woman with a coy smile, is embedded with Golden rectangles. The sketch, An Old Man, has many rectangles on it. Through the Vitruvian Man, Vinci is trying to show the perfection of human body in all divine proportion. The Golden triangles and stars (pentagrams) are used in the composition of several famous paintings including The Holy Family, by Michelangelo and The Crucifixion by Raphael. Salvador Dali used the Golden rectangle as the frame for The Sacrament of the Last Supper. The statue of Athena in ancient Greece and the sculpture of Apollo of the "Belvedere" illustrate the use of Golden ratio. It is present in prolific number in portraits, paintings of Christian God and sculptures during renaissance epoch.

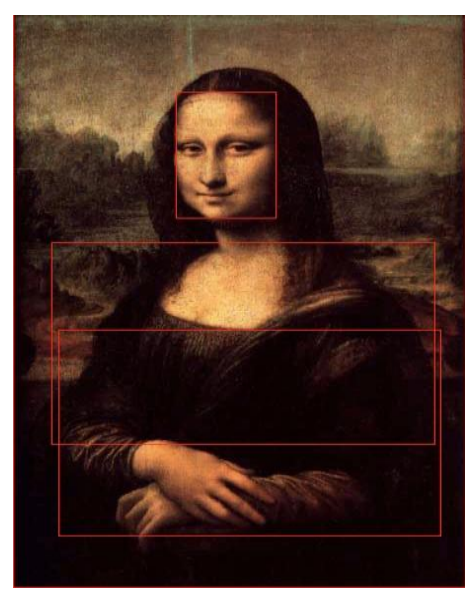

Fig. 13: The Golden Ratio in Art (cuip.uchicago.edu/ dlnarain/golden/activity3.htm)

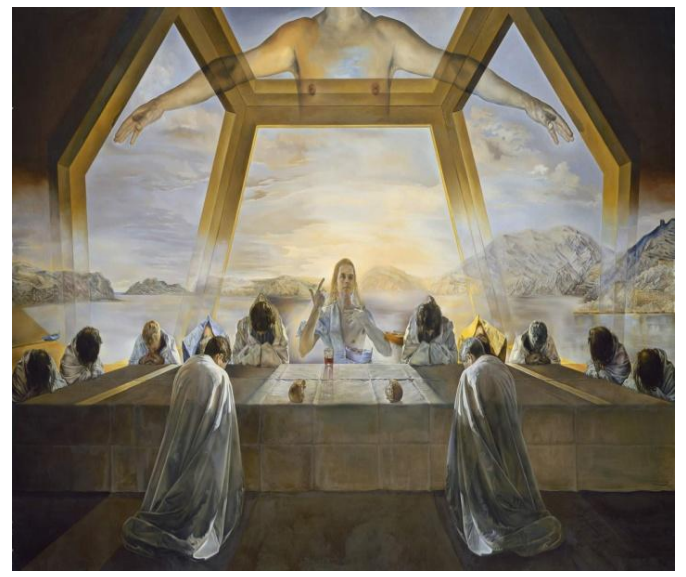

Fig. 14: The Sacrament of the Last Supper

\section{Conclusion}

Advanced mathematical concepts paradoxically lose some relations to the physical world from which they are derived. The intensely scrutinized complex numbers are drawn from the real numbers; yet do not conform to the experience of real numbers to the real world. Mathematics, 
like art stands alone as an object of free perception. Since art is not obliged to make reference to outside world, mathematics is also motivated by wonder and imagination. "The great questions of mathematics- the kind that draw people to math in the first place are called great not because they may lead to applications, but because they captivate the imagination. They inspire wonder and delight. One could say they are beautiful" [5]. The closer the dimensions of the face fit to the Golden ratio, the more beautiful the person is perceived by others. The architects explored connection between geometrical design and artistic beauty when incorporating the Golden ratio into their construction. In modern times, buildings such as the United Nations Headquarters in New York City (having three Golden rectangles) and the CN Tower in Toronto have Golden ratios in their design. Golden ratio is not just lofty mathematical theory; it shows up all the time in the real world. Likewise, graphic designer can use Fibonacci sequence as a general guideline and creative tool to make the design perfect. Designers can incorporate Golden ratio to crop photos or images into Golden rectangle or Golden spiral for the shot's composition, logo design, layout etc. These days' designers are privileged with modern technical apps to employ as the best tools to help them incorporate Golden ratio in their work, such as Atrise Golden section, Golden calipers, Golden ratio app, Golden ratio typography app, Phi calculator etc. People may argue that the Golden ratio probably does not have any mystical powers of beauty drawn from archetypal fabric of the transcendental world. But it is more likely that this ubiquitous pattern has some aesthetically appealing properties suggesting a sense of natural balance and visual harmony.

\section{References}

[1] Craft D (n.d.), The Golden Ratio and Sacred Geometry, Doug Craft Fine Art Home, $<\mathrm{http}: / /$ www.dougcraftfinearts.com/SacredGeometry.htm $>$ Sep, 2012,

[2] Dimare C and Susmitha S (n.d.), The Golden Appeal. Http://www.scientiareview.org/pdfs/199.pdf A Golden Appeal. Massachusetts Academy of Math and Science, Web. Sep 26, 2012,<http://www.scientiareview.org/pdfs/199.pdf A golden Appeal $>$.

[3] Frayling C, Frayling H and Van Der Meer R (1992), The Art Pack. New York: Alfred A. Knopf publishing, USA.

[4] Jensen HJ (2002), Mathematics and Painting, Interdisciplinary Science Reviews, 27(1) : 45-49.

[5] Jones R (2002), Stern Perfection; Mathematics as a Fine Art, Catalyst Special Edition, Brown University, Retrieved from /www. People. Carleton.edu/ rfjones

[6] Narain DL (2012), Teaching Guide. University of Chicago, $<$ http://cuip.uchicago.edu/ dlnarain/golden/teaching_guide.htm $>$.

[7] Parveen, N (n.d.), The Golden Ratio: A Greek History." What Is the Golden Ratio?, The Department of Mathematics and Science Education, The University of Georgia, n.d. Web. 9 Aug. 2012, <http://jwilson.coe.uga.edu/EMAT6680/Parveen/Greek_History.htm>.

[8] Stakhov A and Sluchenkova A (2001), Museum of Harmony and the Golden Section: Mathematical connections in nature, science and art, Vinnitsa: <http://www.goldenmuseum. com> ITI.

[9] http://jwilson.coe.uga.edu/EMT668/EMAT6680.2000/Obara/Emat6690/Golden\%20

Ratio/golden.html (accessed October 4, 2010). 